\title{
Review of Quench Performance of LHC Main Superconducting Magnets
}

\author{
P. Pugnat and A. Siemko
}

\begin{abstract}
The regular lattice of the Large Hadron Collider (LHC) will make use of more than 1600 main magnets and about 7600 corrector magnets, all superconducting and working in pressurized superfluid helium bath. This complex magnet system will fill more than $20 \mathrm{~km}$ of the LHC underground tunnel. In this paper an overview of the cold test program and quality assurance plan to qualify all LHC superconducting magnets will be presented. The quench training performance of more than 1100 LHC main dipoles and about 300 main quadrupoles, cold tested to date, will be reviewed. From these results an estimate of the number of quenches that will be required to start operation of the whole machine at nominal energy will be discussed. The energy level at which the machine could be operated at the early phase of the commissioning without being disturbed by training quenches will be addressed. The LHC magnet program required the development of many new tools and techniques for the testing of superconducting magnet coils, magnet protection systems, cryogenics, and instrumentation. This paper will also present a summary of this development work and the results achieved.
\end{abstract}

Index Terms-Cryogenic test station, LHC, memory effect after thermal cycle, superconducting magnets, training quench.

\section{INTRODUCTION}

$\mathbf{T}$ HE Large Hadron Collider presently under construction at CERN is the result of extensive research and development of various technologies, especially those related to superconducting magnets. To maintain protons in orbit at the unprecedented energy level of $7 \mathrm{TeV}$ within the existing $27 \mathrm{~km}$ circumference LEP tunnel, the 1232 LHC main dipoles must provide a magnetic field of $8.33 \mathrm{~T}$. This is achieved by using $\mathrm{Nb}$-Ti superconducting coils cooled by superfluid helium and a proper mechanical design to sustain the resulting Lorentz force [1]. When the LHC project was approved on the 16 December 1994 by the CERN council [2], the construction and the mass production of the superconducting main magnets were still an issue to be mastered. Today, coming to its end, the mass production of all LHC superconducting magnets can be considered as a great achievement. The quench performance of the main superconducting dipoles (MB) and quadrupoles (MQ) even reached an unprecedented level if one compares results to the performance of main magnets produced at the end of the prototyping phase [3]. Obviously, the LHC superconducting magnet program has profited from the research and development of Fermilab, HERA, SSC and RHIC magnets. It has also benefited from the achievement

Manuscript received August 29, 2006.

The authors are with the European Laboratory for Nuclear Research, CERN, Geneva, CH-1211, Switzerland (e-mail: Pierre.Pugnat@cern.ch).

Digital Object Identifier 10.1109/TASC.2007.898501 of state-of-the-art technology for the $\mathrm{Nb}$-Ti superconducting cables, which was possible thanks to the fruitful collaboration between CERN, Institutes and companies in Europe and world wide, involved since the prototyping phase in the magnet production.

The LHC main superconducting magnets are delivered to CERN in the so-called cold mass state for the operations of cryostating and preparation for the cold tests. When the final acceptance of the magnets is given after the cold tests, they are configured for the machine and stored before being installed in the LHC tunnel at the optimum slot defined by the Magnet Evaluation Board (MEB) [4].

In this review article, the cold test program which is one of the key steps for the quality assurance control of all LHC superconducting magnets will be firstly presented together with its main objectives. The Superconducting Magnet Test Plant (SMTP), specially built at CERN to test all LHC superconducting magnets will be briefly described, jointly with the major hardware and software tools developed. An overview of cold test results will be given, including a brief statistics of all nonconformities detected on test benches as well as highlights of some findings which gave rise to feedback to magnet production lines. The quench training performance of about $1100 \mathrm{LHC}$ main dipoles and 300 main quadrupoles analysed to date will be presented. From these results, including an investigation of the training quench retention after a thermal cycle, the expected performance of the LHC at its earliest running phase will be deduced when beam loss effects can be neglected.

\section{Overview of the Cold Test Program}

\section{A. Objectives}

During the first international review of series tests of LHC lattice magnets held in July 2000 [5], the review board recommended to consider the cold series tests as mandatory in order to guaranty the accelerator reliability and performance. It specifically stressed the importance of tests of all components related to magnet protection in conditions as close as possible to machine operation. Reaching the required test efficiency was considered as a challenge in view of existing planning. The board also strongly supported the decision to construct and fully equip 12 test benches for the cold tests.

To obtain a safe guaranty of the electrical integrity of the main superconducting magnets, it was considered necessary to quench each of them at least two times at or above the nominal current equal to $11850 \mathrm{~A}$. Only high current quenches provide conditions close to known failure modes of superconducting magnet with relevant Lorenz force and voltages which develop in presence of gaseous helium. In addition, to assure the LHC 


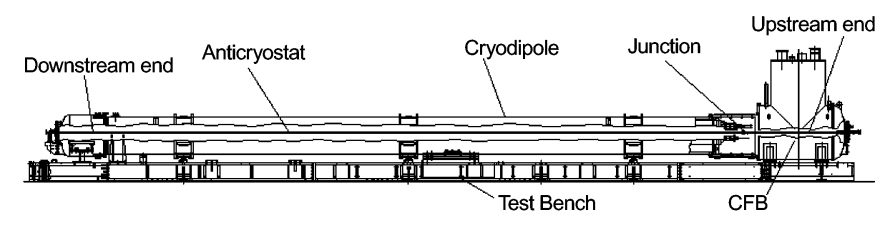

Fig. 1. Layout of a cold test bench with a cryodipole equipped with anticryostats and connected to its Cryogenic Feed Box (CFB). The overall length is equal to $19.6 \mathrm{~m}$.

operation at nominal energy, criteria based on the magnet quench performance were considered since the beginning of the elaboration of the cold test program.

\section{B. The Streamlined Cold Test Program}

The result oriented cold test program of MB and MQ was reviewed and streamlined in 2003 during two internal reviews at CERN to accommodate constraints coming from the LHC planning, the test duration and the capacity of the test stations [6]. As a result, the quench performance evaluation was first based on a two quench criterion with a threshold at $12 \mathrm{kA} \mathrm{[7].}$ An extended test after a Thermal Cycle (TC) was executed for cryomagnets that neither satisfied the two quench criterion nor reached $9 \mathrm{~T}$ after 8 quenches. In 2005, a three quench criterion was finally settled [8] with a threshold at $12.25 \mathrm{kA}$ and the same rule to perform additional training quenches after a TC.

The test program also contains several electrical checks and measurements to ensure the continuity of each circuit, the proper insulation between different circuits and between each circuit and ground. The adopted strategy consists of performing electrical tests after each step that is judged as critical, namely quench tests, cool down and warm-up phases.

\section{The Superconducting Magnet Test Plant at CERN}

\section{A. Infrastructure and Hardware}

The CERN Superconducting Magnet Test Plant (SMTP) contains 12 fully equipped test benches arranged in 6 clusters which can run independently [9]. The layout of a MB installed on a bench is given in Fig. 1. Each bench is composed of a Cryogenics Feed Box (CFB) with its control system, a mechanical support structure and instrumentation racks containing mostly the quench detectors, the magnet protection electronics with the quench heater power supplies, interlocks and two signal acquisition systems $(5-50 \mathrm{kHz}$ and 1-1000 Hz). Racks with noise sensitive instrumentation, such as the one used for magnetic measurements are separated from the main ones. Both benches of each cluster share power converters as well as instrumentation racks. To allow the commutation of the power supplies between two neighboring benches, namely the main one (16 $\mathrm{V} / 14 \mathrm{kA}$ with an external polarity inverter) and the smaller ones (mostly $\pm 600 \mathrm{~A} / \pm 10 \mathrm{~V}$ and $\pm 60 \mathrm{~A} / \pm 8 \mathrm{~V}$ ) used to power corrector magnets as well as for routing the magnet instrumentation wires, special commutation systems were integrated inside each cluster.

A special study was devoted to the electrical connections of the MB and MQ inside the CFB which should be reliable and easily dismountable. The solution retained was based on double overlap joints soldered with indium [10]. To avoid the premature wearing of the superconducting cables of the CFB current lead terminals, bulk silver was deposited electrochemically on each of them.

The tests of the electrical insulation of each circuit are performed with specially developed mobile racks containing a high voltage tester, a relay box, a PLC and a workstation interfacing the hardware with operators and acquiring the data before to send them via a wireless link to a database.

One of the key tools for the quench analysis is the so-called quench antenna, a concept initiated at CERN by J. Krzywinski [11], which allows precise localizations of the quench start position, a key point in the diagnostic of the quench origin. Two different types of quench localization shafts were developed for the series tests. The 15-meter long shafts made of 13 identical segments were designed for magnetic measurements [12] and were applied for a global localization of quench starts, whereas $1 \mathrm{~m}$ long Local Quench Antennas (LQA) with 11 segments for a more precise localization [13], [14].

Quench antennas operate at room temperature and at atmospheric pressure while magnet apertures are cold bores thermalised with the cold mass usually at $\mathrm{T}=1.9 \mathrm{~K}$. Dedicated "warm bore anticryostats" were developed for the use of quench antennas and magnetic field measurement equipment. These anticryostats consist of two concentric, thermally insulated pipes fitted with electrical heaters and covering all magnet length [15].

\section{B. Software}

To ensure the traceability of all instrumentation and equipment used for each magnet test, the so-called Hardware Recognition System was developed, based on barcode identification [16]. This system is also used during the test operation to extract automatically the equipment settings from a database and generate measurement configuration files as a function of the type of test to be performed.

The execution of the test sequences for each magnet type is driven by the so-called TestMaster, software specially developed in Labview to assist and control the operation of the series tests [17]. It also automatically manages the running of various software applications involved during the cold tests and preparatory phases.

The retained strategy for the data analysis was to store all raw data to allow a complete traceability of test results as well as the possibility of an iterative analysis with improved software versions. For each test type a dedicated software tool was developed to provide automatic "online" data analysis. The Automatic Quench Analysis (AQA) software contains most of the experience gained during the prototype, the preseries and the series phases. One of the main roles of AQA is to trigger alarms or warnings in case of abnormal signals to allow dedicated investigation when required. It also ensures a first validation of the results at the test operator level. Dedicated algorithms were developed such as the one to recognize automatically the beginning of the quench from the shape of voltage signals. This is used for a precise estimate of the quench load, expressed with MIITs, i.e. the time integral of the square of the current variation, which allows an estimate of the hot spot temperature inside the magnet coil during each quench. AQA also determines for 
TABLE I

OVERVIEW OF THE NC DETECTED AT CERN ON 1108 CRYODIPOLES

\begin{tabular}{lcc}
\hline \hline NC Type & $\begin{array}{c}\text { Absolute Number } \\
\text { of NC }\end{array}$ & $\begin{array}{c}\text { Average Number of } \\
\text { NC by MB }\end{array}$ \\
\hline Mechanical & 1039 & 0.94 \\
Electrical & 489 & 0.44 \\
Quench Performance & 91 & 0.08 \\
Other & 183 & 0.17 \\
Total & $\mathbf{1 8 0 2}$ & $\mathbf{1 . 6 3}$ \\
\hline \hline
\end{tabular}

MB the values of the resistance of connections of the cold protection bypass diodes when the dedicated provoked quench is performed to trigger the commutation of the diode.

\section{Cold Test Results}

\section{A. Generalities Concerning the Quality Assurance Control}

The quality assurance policy of the LHC project is described in [18] from the overall project organization down to the level of traceability and documentation like procedures and Non-Conformities (NC). To ensure that all $\mathrm{NC}$ were identified during cold tests and preparatory phases before being properly treated, it was necessary to organize the complete traceability of test results as well as to settle four independent lines for the quality control. The first line was taken care by the operators executing the tests and measurements. The second one was assured by quality managers who, on a weekly basis, checked the completeness and the conformity of all cold tests and measurement data. The third line was assured by project engineers who assessed the performance and the conformity of each magnet. The fourth line of quality control was executed by software tools with iterative analysis processes allowing also the discovery and investigation of unidentified factual observations (UFO).

\section{B. Overview of the Non-Conformities Detected}

1) Case of Cryodipoles: In the beginning of August 2006, a total of about 6406 Non-Conformities (NC) concerning 1154 cryodipoles was indexed. Among those $\mathrm{NC}, \sim 60$ were still unresolved at that time. The NC detected at CERN on 1108 cryodipoles are listed in Table I as a function of their main characteristics.

The average number of each NC type by cryodipole is stable since about half of the production. The proportion of MB with low quench performance and submitted to a thermal cycle for extended tests is about $11.5 \%$ whereas only $\sim 1.4 \%$ were rejected for insufficient quench performance. Around $90 \%$ of the electrical NC of Table I was discovered during the cold tests or preparatory phases on the test benches among which around $10 \%$ was due to measurement errors of the electrical continuity at warm conditions.

2) Case of Short Straight Sections: The cold masses of the arc Short Straight Section (SSS) contain the MQ and various corrector magnets that are in general all powered during the cold tests. The average number of electrical NC per SSS detected during cold tests or preparatory phases is around 0.76, i.e. $\sim 1.5$ time larger than for cryodipoles (Table I). This comes from the larger number of electrical problems detected mostly because of the higher complexity of the electrical circuits in SSS cold masses with respect to the one of MB. In average, about $8.2 \%$ of MQ exhibited poor quench performance and were submitted to a thermal cycle for extended tests whereas only one was rejected.

\section{Highlight of Some Findings}

1) Quench Localization: One of the first relevant results obtained with the long quench localization shafts was to point out that, when the design structure of the LHC main dipoles was changed from 5-blocks to 6-blocks in the prototyping phase, the major part of quench starts, and hence the problem of the mechanical stability of the coil winding, moved from straight parts to magnet ends [19]. This diagnostic allowed focusing the efforts during the beginning of the magnet production to the precise shaping of end spacers in pole heads. Even if more than $90 \%$ of training quenches originated in magnet ends for MB, the quench performance reached the acceptable level for the LHC.

For the quenches performed at the conductor limit around $4.4 \mathrm{~K}$, long quench antennas allowed to detect and to identify some degradation of the conductor performance as well as faulty electrical connections between the superconducting cables of the inner and the outer layers of MB [20]. A marginal problem limiting the quench performance at $1.9 \mathrm{~K}$ of a $\mathrm{MB}$ at about $71 \%$ of the nominal current was also encountered and with the axial localization provided by the quench antennas, a conductor cold weld could be found.

Results obtained with Local Quench Antennas (LQA) improved significantly the localization of the quench start and the measurement of the quench velocity. For example, it was found on several MB that most of the training quenches originated in ends are localized at the beginning of the bending of some particular turns [13], [14].

This brief overview concerning the quench localization and the identification of assembly faults would not be complete without emphasizing that the origin of the poor training quench performance of rejected MB could not every time be clearly identified. In real coils tiny mechanical defects, not always identified, can be responsible of the degradation of magnet quench performance especially for the MB of the LHC for which the enthalpy margin of the conductor was constrained to the unprecedented low value of $\sim 0.9 \mathrm{~mJ} / \mathrm{cm}^{3}$.

2) Statistical Analysis of Spikes: Premature training quenches of MB and MQ usually arise because of conductor motions under the action of Lorentz forces. These mechanical events induce transient energy released within the coil as it is energized. In general they also produce a rapid variation of the voltages across quench antennas and different parts of the magnet coil called spikes. To improve the diagnostic concerning the mechanical stability of the coil winding the statistical analysis methods were developed to treat the spatial localization and the time occurrence of spikes as well as their distribution in amplitude and energy [14], [21], [22]. The analogy between the occurrence of spikes inside superconducting magnets and the seismic behaviour of the earth was considered as a starting point as similar power law was found for the distribution of the events as a function of their amplitude [14]. It brought qualitative inputs for a better understanding of the training mechanism of non-impregnated superconducting magnets. 
Even if encouraging results were obtained concerning for example the prediction of some quenches from the stochastic analysis of spikes, this study is still ongoing at the fundamental level of the dynamics of complex systems.

3) Failures of the Electrical Integrity: A failure mode affecting the insulation and continuity of the quench heater $(\mathrm{QH})$ circuits of $\mathrm{MB}$, was detected on test benches at the beginning of the production [23]. The problem was identified inside the cold mass around the so-called omega piece assuring the connection in series of two heater strips. This failure originated from uncontrolled local over-stress and resulting damage of end spacers due to inappropriate shimming in the coil. To intercept as early as possible MB with this defect, the combination of electrical insulation and continuity tests with the powering of quench heaters at relevant parameters was implemented in industry at warm conditions with the proper analysis tools [23].

Various other types of electrical insulation failures were also detected. Few cases of inter-turn short circuits were experienced on the test benches during quenches of MB. One of them was particularly destructive as a hole was melted out in the $2 \mathrm{~mm}$ thick stainless steel cold bore tube which provoked the rupture of the vacuum barrier inside one aperture and the projection of He gas. The estimate of the maximum local temperature during this event gives a value around $3000^{\circ} \mathrm{C}$ as one ceramic pipe of a quench antenna showed local signs of partial melting whereas just after the cryostat of the $\mathrm{MB}$ was found at a temperature below $-50^{\circ} \mathrm{C}$.

Few breakdowns of the insulation or abnormal leakage current were found between main circuits or auxiliary ones and the ground. This type of fault, affecting more often SSS cold masses, was in general not easy to diagnose as it could also be induced by humidity problems around the current leads of the cryogenic feed boxes.

One of the electrical weakest points identified during the cold tests inside cryomagnets of SSS or MB type is the Instrumentation Feedthrough System (IFS) [24]. It cumulates during the powering of quench heaters the constraints of high voltage between circuits (up to $900 \mathrm{~V}$ ) and high current in half of them (up to $70 \mathrm{~A}$ ) with time constant around $200 \mathrm{~ms}$ in a tight environment which contains warm helium gas. It has received particular attentions as it has revealed the larger proportion of electrical failures on the test benches affecting both properties of insulation and continuity of circuits.

4) Diode Contact Resistance: In autumn 2003, a major electrical fault concerning the continuity of all cold diode circuits was detected [25], [26]. The contact resistances of all diode bus-bars connections were found too high. Corrective actions were undertaken at CERN and in all production lines in industry, including the revision of the assembling procedures and the implementation of a tight quality control based on measurements of the resistance of the diode bus bars connections at warm and at cold conditions.

\section{Training Quench Performance}

1) Case of $M B$ : The histogram of the cold tested MB as a function of the number of training quenches required to reach the nominal field of the LHC is shown in Fig. 2. Before the

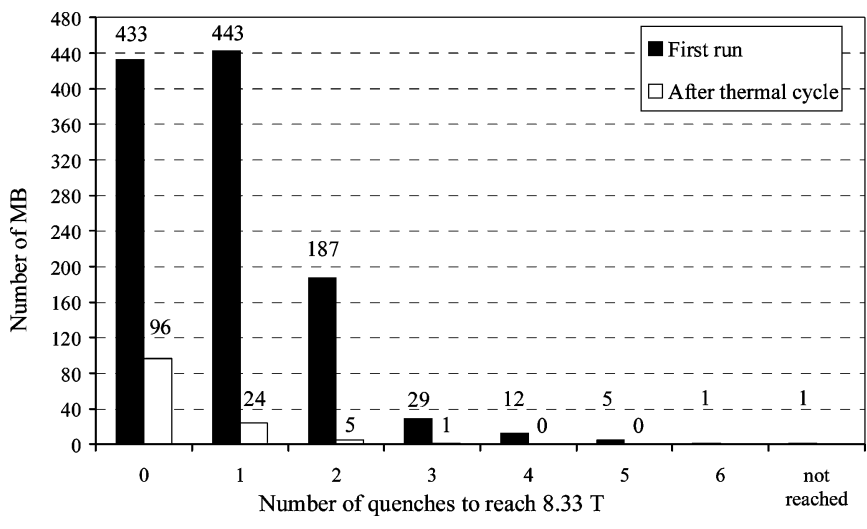

Fig. 2. Histogram of the $1111 \mathrm{MB}$ cold tested to date and produced by the three European manufacturers as a function of the number of training quenches after the first and second cool-down. The additional thermal cycle was performed on the $128 \mathrm{MB}$ with the weakest quench performance.

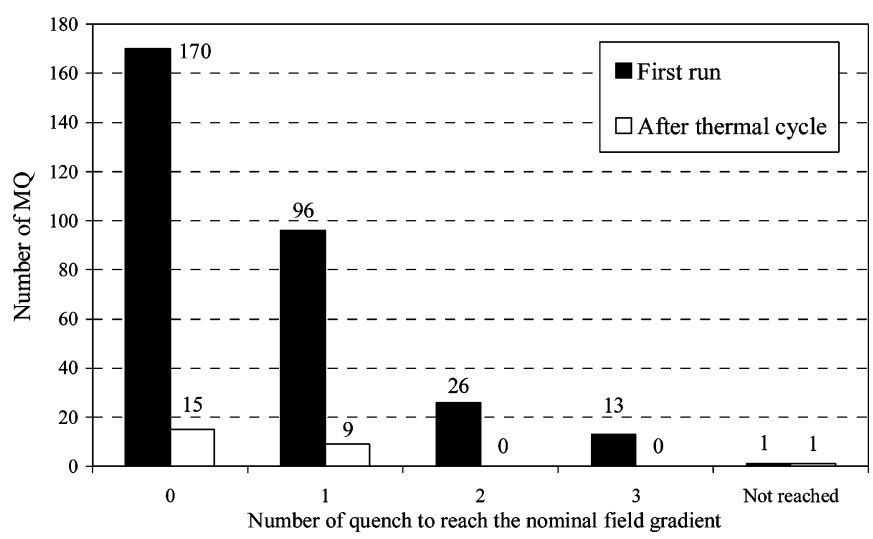

Fig. 3. Histogram of the $306 \mathrm{MQ}$ cold tested to date as a function of the number of training quenches occurring after the first and second cool-down. The additional thermal cycle was performed on the $25 \mathrm{MQ}$ with the weakest quench performance.

thermal cycle (TC), about $39 \%$ of $\mathrm{MB}$ reached the nominal field without training quench during their first powering. After a TC performed on $\sim 11.5 \%$ of MB, mostly for reason of weak quench performance, this proportion reached $\sim 75 \%$ (Fig. 2) i.e. $\sim 25 \%$ of $\mathrm{MB}$ required at least one training quench to reach the nominal field equal to $8.33 \mathrm{~T}$.

All MB that did not reach the nominal field were rejected and repaired in industry before being re-tested at cold. From the simplest extrapolation of the results of Fig. 2, assuming no detraining effect and that all MB submitted to a TC will not quench in the tunnel, the number of quenches that may occur during the first powering cycles up to nominal field is around 330. As the eight octants of the LHC will be powered independently, one has to consider about 40 quenches by octant but this corresponds to a worst case scenario with a low probability of occurrence as it is based on a biased statistics coming from the sample of the weakest MB for which a TC was performed. This estimate will be reassessed in paragraph $\mathrm{V}$.

2) Case of $M Q$ : The histogram of the cold tested MQ as a function of the number of training quenches required to reach the nominal field gradient, assumed to be equal to $223 \mathrm{~T} / \mathrm{m}$ i.e. $11850 \mathrm{~A}$, is given in Fig. 3. Before the thermal cycle, about $56 \%$ 
TABLE II

StATISTICS PER MB RELATED TO THE NUMBER OF QUENCHES TO REACH THE NOMINAL FIELD OF 8.33 T $(11850 \mathrm{~A})$

\begin{tabular}{lcc}
\hline \hline Sample & $\{$ MB with TC $\}$ & $\{$ MB without TC $\}$ \\
\hline Average Number before TC & 1.82 & 1 \\
Standard Deviation & 1.35 & 1 \\
\hline Average Number after TC & 0.33 & $0.181^{*}$ \\
Standard Deviation & 0.78 & $0.58^{* *}$ \\
\hline Population & 128 & 983 \\
\hline * assuming the same reduction of $82 \%$ after TC as for $\{\mathrm{MB}$ with TC $\}$ \\
$* *$ assuming the same reduction of $42 \%$ after TC as for $\{\mathrm{MB}$ with TC $\}$
\end{tabular}

of MQ reached the nominal field gradient during their first powering without quench. After a TC performed on only $25 \mathrm{MQ}$ because of a weak quench performance, this proportion becomes $15 / 25$. The training retention of MQ seems to be smaller with respect to MB but the statistics is less even if more cases could be considered since the previous study [27]. The MQ magnet which did not reach the nominal field gradient will be repaired.

\section{EXPECTED LHC Quench PeRFoRMANCE DuRING FIRST POWERING CYCLES}

\section{A. Case of $M B$}

1) Estimate of the Number of Quenches by Octant to Reach the Nominal Field of the LHC: The obvious consequence of the applied test programs is to introduce a statistical bias for the sample $\{\mathrm{MB}$ with $\mathrm{TC}\}$ that must be corrected for a reliable training quench prediction for the machine. A simple method was proposed [27] and is summarized in Table II together with the main statistical parameters used and deduced.

From the results of Table II, the average number of quenches that can be expected to occur below the nominal field in the machine is equal to $25 \pm 6$ by octant at $\pm 2 \sigma$. The implicit assumptions made for this estimate are listed below before being commented:

i) No faulty MB will be accepted;

ii) No drift in quench performance for future $\mathrm{MB}$;

iii) No quench is expected for MB submitted to a TC on test benches;

iv) No long time relaxation effect of the quench performance of the trained magnets;

v) No detraining quenches.

The hypothesis i) does not require further development as the acceptance of the remaining MB will be based on the same criteria. For ii), as one of the firm producing the MB with quench performance above the average had already delivered all its production, a slight drift may occur and should be looked at when all MB will be cold tested at the end of 2006. Concerning iii), all MB for which at least two TC were performed, reached $8.33 \mathrm{~T}$ without quench after the third cool-down. For iv), the two targeted MB for the study of the long term stability did not reveal a significant drift of the quench performance after about 1 year of storage [28]. The last hypothesis v) is the most questionable and an upper estimate of the number of quench due to a detraining effect can be given. The probability to have a detraining effect around the nominal field after a TC was found to be $\sim 4 \%$ from the sample $\{\mathrm{MB}$ with $\mathrm{TC}\}$. If it is assumed that when a $\mathrm{MB}$ quenches, in average its two neighbours will also quench because of the quench-back induced by the warm He gas, then the maximum number of additional quenches due to the detraining effect is of the order of $0.04 \times 3 \times 25<5$ by octant, i.e. a value larger than the estimated standard error. The detraining effect will be a more serious problem when the magnets will be pushed to current value much higher than the nominal one.

2) Quench Probability Versus Current for MB: From the cumulative statistics of $\mathrm{MB}$, the probability to have a MB quench can be estimated as a function of the magnetic field [27]. Preliminary results give that at the probability level of few \%, training quenches will start typically at current value in $\mathrm{MB}$ of about $11 \mathrm{kA}(6.5 \mathrm{TeV})$.

\section{B. Case of $M Q$}

Estimate of the Number of Quenches by Octant to Reach the Nominal Field Gradient and Quench Probability Versus Current: The same statistical approach as for MB was applied for MQ. The number of quenches that can be expected to occur below the nominal current equal to $11850 \mathrm{~A}$, is found to be around 5 by octant, a slightly lower value with respect to the previous one obtained from a study using a more reduced statistics [27]. An estimate of the standard error gives around 2 training quenches by octant. Both values obtained have to be reassessed with results of all MQ once cold tested to profit of the overall statistics, especially for the training retention effect after a thermal cycle. Concerning the quench probability versus the current level of MQ, a similar value than for MB was obtained as a first estimate [27].

\section{CONCLUSION}

The cold tests of all LHC superconducting magnets are coming to an end and have required an important effort within a tight schedule in the construction and operation at CERN of a complex superconducting magnet test plant. The next challenging phases of the LHC are the hardware commissioning of all interconnected magnets as well as the commissioning of the overall machine with beams.

To reach the LHC nominal beam energy of $7 \mathrm{TeV}$ for the first time will imply to cope with training quenches of $\mathrm{MB}, \mathrm{MQ}$ as well as of other superconducting magnets. As a first estimate from present data, 25-30 \pm 6 and $5 \pm 4$ training quenches by octant respectively for MB and MQ are expected. The uncertainties are given for both magnet types at $\pm 2 \sigma$ whereas the systematic error for MB is related to possible detraining effect. At the level of probability of few \%, limited number of training quenches will start typically at current value in $\mathrm{MB}$ of about $11 \mathrm{kA}(6.5 \mathrm{TeV})$ and at a similar level for MQ. Other types of quenches are also expected to occur during the starting and the running of the LHC, such as the ones due to bad electrical connections, cryogenic problems and beam losses. Dedicated investigations are now going on to reassess the possible LHC limitations in presence of beam losses as a function of each superconducting magnet type and their position in the ring. 


\section{ACKNOWLEDGMENT}

The authors would like to thank all members of the SMTP teams for the production and the validation of the data, N. Smirnov and M. Pojer for regular update of the statistical results of main superconducting magnets, and D. Tommasini for providing the data of Table I.

\section{REFERENCES}

[1] The LHC Study Group, "The Large Hadron Collider Design Report," CERN-2004-003, June 4, 2004, vol. I [Online]. Available: http://www.ab-div.web.cern.ch/ab-div/Publications/LHC-DesignReport.html

[2] CERN/2075/Final, unpublished.

[3] L. Bottura et al., "Performance of the LHC final prototype and first pre-series superconducting dipole magnets," IEEE Trans. Appl. Sup. vol. 12, pp. 211-214, March 2002 [Online]. Available: http:// www.doc.cern.ch/archive/electronic/cern/preprints/lhc/lhc-project-report-556.pdf

[4] L. Bottura et al., "The Magnet Evaluation Board," CERN LHC Project Workshop-"Chamonix XIV", February 2005 [Online]. Available: http://www.ab-div.web.cern.ch/abdiv/Conferences/Chamonix/chamx2005/PAPERS/7_08.pdf

[5] L. Walckiers et al., "Review at CERN of Series Tests of LHC Lattice Magnets: Summary of the Recommendations of the Board," Doc LHCMTA, Sept. 21, 2000, unpublished.

[6] "Review of Reception Tests of LHC Dipole Cryomagnets," CERNEDMS Id 399278, July 2003, unpublished, "Review of Reception Tests of LHC Cryomagnets," CERN-EDMS Id 427483, December 2003, unpublished.

[7] Minutes of the CERN Internal Meeting, MARIC 2004-85, unpublished.

[8] Minutes of the CERN Internal Meeting, MARIC 2005-112, unpublished.

[9] A. Siemko et al., "Quench performance and field quality of the LHC preseries superconducting dipoles," IEEE Trans. Appl. Sup. vol. 14, no. 2, pp. 165-168, 2004 [Online]. Available: http://www.doc.cern.ch/ archive/electronic/cern/preprints/lhc/lhc-project-report-707.pdf

[10] F. Beauvais et al., "Clamped JOINTS Investigation: An Alternative Solution to Connect Long Dipole Magnets to the CFU at SM-18 (Part 2)," Internal Note CERN-LHC-MTA-99-07, unpublished. [Online]. Available: https://www.edms.cern.ch/file/356797/1/mta-in-99-074.pdf

[11] D. Leroy et al., "Quench observation in LHC superconducting one meter long dipole models by field perturbation measurements," IEEE Trans. Appl. Sup. vol. 3, pp. 781-784, 1993 [Online]. Available: http:// www.ccdb4fs.kek.jp/cgi-bin/img/allpdf?199301021

[12] J. Billan et al., "Twin rotating coils for cold magnetic measurements of $15 \mathrm{~m}$ long LHC dipoles," IEEE Trans. Appl. Sup., vol. 10, no. 1, pp. 1422-1426, March 2000.

[13] M. Calvi, Ph.-D Thesis, University of Geneva, December 2004, unpublished.

[14] M. Calvi et al., "Improved quench localization and quench propagation velocity measurements in the LHC superconducting dipole magnets," IEEE Trans. Appl. Sup. vol. 15, no. 2, pp. 1209-1212, 2005 [Online]. Available: http://www.doc.cern.ch/archive/electronic/cern/preprints/lhc/lhc-project-report-809.pdf
[15] O. Dunkel, P. Legrand, and P. Sievers, in AIP Conferences Proceedings, 2004, vol. 710, no. 1, p. 494 [Online]. Available: http:// www.doc.cern.ch/archive/electronic/cern/preprints/lhc/lhc-project-report-685.pdf

[16] A. Raimondo et al., "The hardware recognition system for the SM18 LHC cryomagnets test benches," in Proceedings of the International Conference on Accelerator and Large Experimental Physics Control System, Gyeongju, Korea, October 13-17, 2003 [Online]. Available: http://www.ab-dep-co-ma.web.cern.ch/ab-dep-co-ma/docs/paperHWREC.pdf

[17] A. Rijllart et al., "Orchestrating the measurements of the twelve magnet test benches," in Proceedings of European Particle Accelerator Conference, EPAC 2002, Paris, France [Online]. Available: http://www.abdep-co-ma.web.cern.ch/ab-dep-co-ma/docs/MOPDO013.pdf

[18] P. Faugeras, "Quality Assurance Policy and Project Organisation," CERN-EDMS Id 103544, unpublished. [Online]. Available: http:// www.lhc-proj-qawg.web.cern.ch/lhc-proj-qawg/LHCQAP/\#100

[19] L. Bottura, P. Pugnat, A. Siemko, J. Vlogaert, and C. Wyss, "Performance of the LHC final design, full-scale superconducting dipole prototypes," IEEE Trans. Appl. Sup. vol. 11, no. 1/2, pp. 1554-1557, 2001 [Online]. Available: http://www.doc.cern.ch/archive/electronic/ cern/preprints/lhc/lhc-project-report-452.pdf

[20] M. Pojer, P. Pugnat, A. Siemko, A. Verweij, and B. Vullierme, "Conductor-limited quenches of LHC superconducting main dipoles," IEEE Trans. Appl. Sup. vol. 15, no. 2/2, pp. 1554-1557, 2005 [Online]. Available: http://www.doc.cern.ch/archive/electronic/cern/preprints/lhc/lhc-project-report-807.pdf

[21] P. Pugnat et al., "Statistical diagnosis method of conductor motions in superconducting magnets to predict their quench performance," IEEE Trans. Appl. Sup. vol. 11, no. 1, pp. 1705-1708, 2001 [Online]. Available: http://www.doc.cern.ch/archive/electronic/cern/preprints/lhc/lhc-project-report-455.pdf

[22] M. Calvi, N. Ponomarev, P. Pugnat, and A. Siemko, "Statistical analysis of conductor motions in LHC superconducting dipole magnets," IEEE Trans. Appl. Sup. vol. 14, no. 2, pp. 223-226, 2004 [Online]. Available: http://www.doc.cern.ch/archive/electronic/cern/preprints/lhc/lhc-project-report-699.pdf

[23] P. Pugnat et al., "Investigation of the Electrical Insulation Problems Developed in the Quench Heater Circuits of the LHC Pre-Series Cryo-Dipole HCLBBR_000-CR002002," Internal Note CERN-LHC-MTA-IN-2002-186 [Online]. Available: https://www.edms.cern.ch/file/339478/1/mta-in-2002-186.pdf

[24] D. Bozzini, "The standard instrumentation feedthrough system for the LHC cryomagnets," IEEE Trans. Appl. Sup. vol. 12, no. 1, pp. 1269-1271, 2002 [Online]. Available: http://www.doc.cern.ch/ archive/electronic/cern/preprints/lhc/lhc-project-report-550.pdf

[25] Minutes 2003-11, CERN EEWG Meetings, unpublished. [Online]. Available: http://hcp.web.cern.ch/lhcp/tcc/powering/eewg/ EEWGMin03.htm

[26] E. Floch, M. Calvi, P. Pugnat, and A. Siemko, "Electrical Resistance at Cold of Diode Busbar Connections-First Results Obtained on LHC Main Dipoles and Preliminary Analysis," Internal Note CERN-AT-MTM-IN-2003-064, unpublished. [Online]. Available: https://www.edms.cern.ch/file/408471/1/AT-MTM-IN-2003-064.pdf

[27] P. Pugnat, "Expected quench Levels of the machine without beam: Starting at 7 TeV?," CERN LHC Project Workshop-"Chamonix XV", February 2006 [Online]. Available: http://www.ab-div.web.cern.ch/abdiv/Conferences/Chamonix/Chamx2006/PAPERS/PP_4_06.pdf

[28] F. Seyvet et al., "Long term stability of the LHC superconducting cryodipoles after outdoor storage," IEEE Trans. Appl. Sup. vol. 16, no. 2, pp. 180-183, 2006 [Online]. Available: http://www.doc.cern.ch/ archive/electronic/cern/preprints/lhc/lhc-project-report-895.pdf 